\title{
Motional Stark Effect Measurements of the Local Magnetic Field in High Temperature Fusion Plasmas
}

\author{
Robert C. Wolf* ${ }^{1}$
}

E-mail: robert.wolflipp.mpg.de

\author{
O. Ford', R. Reimer ${ }^{1}$, A. Bock1, A. Burckhart ${ }^{1}$, A. Dinklage1, J. Hobirk1, J. Howard², \\ M. Reich ${ }^{1}$, J. Stober ${ }^{1}$ \\ ${ }^{1}$ Max Planck Institute for Plasma Physics, 85748 Garching and 17491 Greifswald, Germany, \\ ${ }^{2}$ Plasma Research Laboratory, Australian National University, Canberra, Australia
}

\begin{abstract}
The utilization of the Motional Stark Effect (MSE) experienced by the neutral hydrogen or deuterium injected into magnetically confined high temperature plasmas is a well established technique to infer the internal magnetic field distribution of fusion experiments. The different properties of the Stark multiplet allow inferring, both the magnetic field strength and the orientation of the magnetic field vector. Besides recording the full MSE spectrum, several types of polarimeters have been developed to measure the polarization direction of the Stark line emission. To test physics models of the magnetic field distribution and dynamics the accuracy requirements are quite demanding. In view of these requirements, the capabilities and issues of the different techniques are discussed, including a newly developed Imaging MSE system which has been tested on the ASDEX Upgrade tokamak.
\end{abstract}

First EPs Conference on Plasma Diagnostics - $1^{\text {st }}$ ECPD

14-17 April 2015,

Villa Mondragone , Frascati (Rome) Italy

\section{Introduction}

Understanding magnetic confinement of high temperature fusion plasma, i.e. plasma transport and stability, requires the detailed knowledge of the magnetic field inside the plasma. In stellarators the magnetic field is provided, at least to a large extent, by external magnetic field coils. Nevertheless, at finite $\beta$ plasma currents modify the vacuum magnetic field and as a consequence also the confinement properties. In tokamaks, the poloidal magnetic field generated by the toroidal plasma current distribution forms an essential part of the confining magnetic field. Its intrinsic coupling to the plasma properties such as electrical conductivity or plasma transport makes measurements of the magnetic field distribution an essential tool for understanding tokamak plasmas.

Since the early days of fusion research non-invasive diagnostic techniques to infer the magnetic field measurement inside plasma have been the focus of intensive developments (see e.g. review by Soltwisch ${ }^{1}$ ). The problem with all these techniques is, however, that the first order of the magnetic field distribution is generally not of interest. Typically, the equilibrium current distribution or safety factor profile in a tokamak can be derived from the knowledge of

*Speaker 
the pressure profile and magnetic probes measuring the field outside the plasma. In stellarators the vacuum magnetic field, which already confines the plasma, can be even measured without plasma employing electron beam techniques ${ }^{2}$. However, the interesting details of plasma transport or stability often depend on small deviations from these first order safety factor or rotational transform profiles. Also the diamagnetic reduction of the magnetic field is only of the order of a few percent. To measure these small variations the corresponding technique must offer an appropriate sensitivity and accuracy.

The Motional Stark Effect (MSE) observed on high energy neutrals injected into the plasma turned out to be one of the most powerful methods to measure the magnetic field distribution inside high temperature plasmas. In the older literature, pointing out the diagnostic potential, the Motional Stark Effect is often referred to as translational Stark Effect ${ }^{3,4}$. Prior to that, the effect was described in the context of fast hydrogen atoms entering the plasma through charge exchange with energetic protons ${ }^{5}$. First introduced on the tokamaks PBX-M ${ }^{6}$ and $\mathrm{JET}^{7}$ in 1989, today MSE diagnostics can be found on many fusion devices including tokamaks ${ }^{8,9,10}$ and stellarators (or heliotrons) ${ }^{11,12}$. Employing hydrogen or deuterium neutral beams (or in a few cases on JET also tritium beams), the linear Stark effect generated by the Lorentz electric field, $\boldsymbol{E}_{\boldsymbol{L}}=\boldsymbol{v} \times \boldsymbol{B}$, in the frame of the fast hydrogen moving with the beam velocity $\boldsymbol{v}$ with respect to the background magnetic field $\boldsymbol{B}$ produces a characteristic emission line pattern. MSE diagnostics normally use the Stark-split Balmer- $\alpha$ transition $(n=3 \rightarrow 2)$ which basically consists of nine emission lines with a characteristic wavelength splitting and $\sigma$ - and $\pi$-polarization. The polarization of the line emission and thus the ratio between $\sigma$ - and $\pi$-polarized lines are sensitive to the orientation of $\boldsymbol{E}_{L}$, while the wavelength splitting is determined by its strength. Translating this into a measurement of the magnetic field, the orientation of $\boldsymbol{B}$ can be inferred from the measurement of the polarization or the $\sigma$ - to $\pi$-ratio, while the wavelength splitting contains information about the magnetic field strength. Since the radial electric field in fusion plasmas, $\left|\boldsymbol{E}_{\boldsymbol{r}}\right|$, is typically two orders of magnitude smaller than $\left|\boldsymbol{E}_{\boldsymbol{L}}\right|$, it only has a significant influence on the MSE polarization measurement, provided the angle between $\boldsymbol{E}_{\boldsymbol{r}}$ and $\boldsymbol{E}_{\boldsymbol{L}}$ is large enough.

Many magnetic confinement fusion experiments which are also equipped with a neutral beam injection system employ a multi-channel MSE diagnostic to measure the radial profile of the magnetic field. Most such systems focus on the polarization measurement for the determination of the toroidal current density profile. In a few cases also spectrally resolved MSE diagnostics are used to infer $|\boldsymbol{B}|$ and in some cases also $\boldsymbol{B}$ from the $\sigma$ - to $\pi$-line ratio. Despite the wide distribution of this technique, it still suffers from a number of issues related to the desired accuracy of such measurements. Calibrating MSE diagnostics and, in particular, polarization measurements in a fusion experiment environment is still challenging. In view of the increasing accuracy with which the spectral and polarization information can be measured, many inconsistencies or inaccuracies in the previous analysis become apparent. These include atomic physics effects such as the correct treatment of the population densities of the atomic levels, which determine the line intensities ${ }^{13,14,15}$, or the admixture of the Zeeman Effect to the Stark Effect $^{4,15}$. There is also strong evidence that metallic plasma facing walls result in polarized reflections from the plasma background radiation leading to spurious polarization signals in the MSE diagnostic ${ }^{16}$. 
This paper is organized as follows: First the Motional Stark Effect and its basic features as plasma diagnostic are briefly introduced. Two basic measurement techniques are discussed. The first relies purely on the analysis of wavelength dependent emission distribution of the MSE spectrum. The second employs polarization measurements utilizing the characteristic polarization information contained in the MSE emission. Finally, before concluding the paper, the so-called Imaging MSE diagnostic, which is a special form of polarization measurement, will be presented.

\section{Motional Stark Effect}

The Stark Effect describes the removal of the degeneracy of the atomic levels due to an external electric field superimposed to the atomic Coulomb field. The linear Stark effect only appears if the atomic levels are fully degenerate as it is the case for hydrogen. In the translational or Motional Stark Effect the electric field is caused by the $\boldsymbol{v} \times \boldsymbol{B}$ electric field (Lorentz electric field) appearing in the rest frame of an atom moving with the velocity $v$ through a magnetic field $\boldsymbol{B}$. In fusion research powerful neutral heating beams, using hydrogen or its isotopes deuterium and in a few cases also tritium, or dedicated diagnostic beams with high power densities have facilitated the development of MSE diagnostics. With beam energies of the order of $100 \mathrm{keV}$ and magnetic fields of several Tesla, $\left|\boldsymbol{E}_{\boldsymbol{L}}\right|$ lies in the range of $10^{6}$ to $10^{7} \mathrm{~V} / \mathrm{m}$. In the case of the Balmer- $\alpha \mathrm{n}=3 \rightarrow 2$ transition, the $\mathrm{n}=3$ level splits into five and the $\mathrm{n}=2$ into three energy levels. Altogether the selection rules produce nine distinct transitions which can be measured ${ }^{7}$.

The emitted light originates from the interaction of the neutral beam with the plasma ${ }^{17,18}$. The neutral atoms are excited by collisions with plasma particles and the radiative de-excitation generates the characteristic beam emission. As the neutral beam particles are also ionized by beam-plasma collisions, the beam is successively attenuated when penetrating the plasma. Higher electron densities and longer penetration depths corresponding to larger plasma crosssections or more tangential injection along the toroidal axis of the device result in stronger attenuation. As a consequence the intensity of the beam emission decreases limiting the applicability as a diagnostic. The other parameters determining the beam attenuation are the beam energies, which typically lie in the range between 50 and $500 \mathrm{keV}$, and the energy composition, which in the case of positive ion beam sources consists of three energy species at full, half and third energy.

Fig. 1. Typical setup of a beam emission diagnostic. The beam (yellow) is intersected by a fan of lines of sight (green). The approximate orientation of the magnetic field and the corresponding Lorentz electric field are also shown. The background is a view of the JET vacuum vessel and, on the right hand side, the overlaid picture of the plasma emission in the visible ${ }^{19}$.

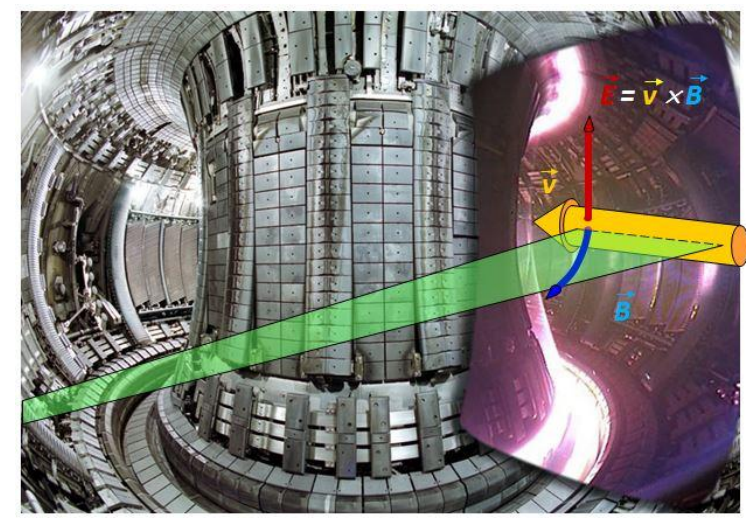


Beam and viewing geometries influence the characteristics of the spectrum and the way the measurement can be analysed. Typical viewing and beam geometries are shown in Fig. 1. To spectrally separate the beam emission from other plasma emission, originating from the same atomic transition, the beams are observed at angles different from $90^{\circ}$. In the example shown, the lines of sight look into the beam resulting in a blue shift of the beam emission spectrum. An example MSE spectrum, measured at $\mathrm{JET}^{18}$, is shown in Fig. 2. With nine MSE lines per energy component, altogether 27 blue-shifted lines are required to describe the spectrum. The spatial resolution of the diagnostic is given by the cross-section of the lines of sight and the volume defined by the lines of sight crossing the beam diameter. Depending on the dimensions of the beam with respect to the plasma dimensions and the variations of the plasma parameters of interest along the line of sight through the beam, the measurement can be regarded as local with some averaging of the plasma parameters along the emission volume. However, in particular in smaller fusion experiments, using relatively large high power heating beams, inversion techniques might become necessary to infer the plasma parameters from the line integrals through the beam diameter. Using a forward model to describe the measured data the line integration can be implemented in a straightforward way ${ }^{20}$. Finally, in some cases also the influence of the neutral beam on the plasma has to be considered. If the beam, which is used to heat and sustain the plasma, is also used for the MSE measurement, this is not an issue. In the case where the beam is only applied for diagnostic purposes, dedicated diagnostic beams with lower power but high power density are required. If they are not available some experiments use short beam blips to minimize the effect of the beam on the plasma ${ }^{21}$.

Fig. 2. Example MSE spectrum using deuterium beams measured at $J E T^{18}$. Clearly visible are the three energy components. Also the deuterium Balmer- $\alpha$ edge emission, the so-called charge exchange "pedestal" and the hightemperature charge exchange line from the interaction of the neutral beam with the core plasma can be seen.

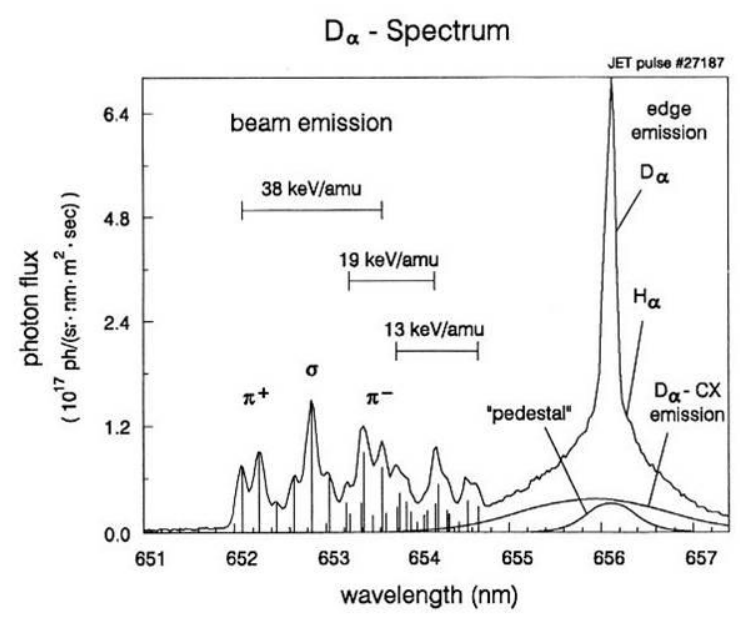

\section{Measurement of spectral properties}

\subsection{Measurement techniques}

As an example the observation system of the ASDEX Upgrade spectroscopic MSE diagnostic is described ${ }^{22}$. Similar systems can be found on many fusion experiments ${ }^{12,18,23,24}$. Near the plasma boundary a mirror is used to reflect the light collected from the neutral beam onto a more radial path. Subsequently a lens system focuses the light onto a fibre bundle which relays the light to a spectrometer which generates the spectra for different radial locations. On 
ASDEX Upgrade a window in front of the mirror protects the mirror against coating from plasma impurities and thus maintains its reflection properties. A dielectric mirror, designed for high reflectivity near the Balmer- $\alpha$ wavelength and for the reflection angles required, guarantees optimal reflection properties also for the differently polarized spectral lines. The beam emission spectra are recorded by a CCD camera. At ASDEX Upgrade the full spectrum including the Balmer- $\alpha$ edge emission is recorded. Since this spectral emission is usually very strong, the centre of the line is blocked out by a thin metal wire which in the exit plane of the spectrometer is positioned exactly at the wavelength of this line. Keeping the wavelength range around the edge emission in the spectrum has the advantage that the charge exchange components of the spectrum reaching below the MSE spectrum and the Doppler shift between MSE energy components and edge emission are better constrained.

\subsection{Spectral properties and analysis}

Each energy component of the Stark multiplet consists of $\sigma$ - and $\pi$-lines (see Fig. 2). The six $\pi$-lines are polarized parallel to the projection of $\boldsymbol{E}_{\boldsymbol{L}}$, while the three $\sigma$-lines are an incoherent superposition of left and right hand elliptically polarized emission. Thus, observed parallel to $\boldsymbol{E}_{\boldsymbol{L}}$ the $\sigma$-emission is unpolarized, observed perpendicular to $\boldsymbol{E}_{\boldsymbol{L}}$ it becomes linear. Inbetween the $\sigma$-emission is linearly polarized with an unpolarized background. The ratio of the $\sigma$ - to $\pi$-emission characteristic is given by $\left(1+\cos ^{2} \Theta\right) / \sin ^{2} \Theta$, where $\Theta$ is the angle between $\boldsymbol{E}_{\boldsymbol{L}}$ and the line of sight. In addition to this emission characteristic line intensities depend on the population densities of the $n=3$ levels and the transition probabilities. The latter can be easily derived from the atomic physics of the Stark effect. However, already early on it was discovered that the assumption of a statistical population of the upper levels does not agree with the observed line ratios ${ }^{18}$. More recent measurements show a clear density dependence of the relative intensities of the Stark multiplet ${ }^{13}$. Theoretical considerations can explain this by a nonstatistical population of the atomic levels involved ${ }^{25,14}$. Even for plasma densities of a few $10^{20} \mathrm{~m}^{-3}$ a statistical population distribution is not achieved. For calibration purposes the beam is often injected into the plasma vessel filled with neutral gas. Also in this case the populations of the $\mathrm{n}=3$ states is actually far from a statistical distribution ${ }^{25}$.

The individual line widths of the Stark multiplet depend on the integration of the emission over the velocity distribution of the neutral beam particles along the line of sight ${ }^{26}$. This results in a dependence on beam and line of sight divergence which basically add up to an effective line width. Other effects which can contribute to the line broadening are variations of the magnetic field over the line of sight and the ripple of the acceleration voltage of the neutral beam injectors ${ }^{27,28}$.

The line splitting contains the information on the magnetic field strength. For the pure Stark effect the line splitting is proportional to $\left|\boldsymbol{E}_{\boldsymbol{L}}\right|$. In an idealized geometry (beam injection perpendicular to the magnetic field) this results in a direct proportionality to the total magnetic field strength $|\boldsymbol{B}|=\left(B_{p o l}{ }^{2}+B_{t o r}\right)^{2 / 2}\left(B_{p o l}\right.$ and $B_{t o r}$ are the poloidal and toroidal magnetic field components, respectively).

Although it was pointed out already in the work by Souw and Uhlenbusch ${ }^{4}$ and later also in the $\mathrm{PhD}$ thesis by $\mathrm{Yuh}^{15}$, the influence of the Zeemann Effect on the spectral properties of the MSE spectrum is usually neglected. Comparing the relevant terms in the Schrödinger 
equation $^{18}$, their ratio $\left(\mu_{B} B\right) /\left(3 / 2 e E_{L} a_{0}\right)=\mu_{B} /\left(3 / 2\right.$ e $\left.v a_{0} \sin \alpha\right)$ (where $\mu_{B}$ is the Bohr magnetron, $a_{0}$ the Bohr radius, $v$ the beam velocity and $\alpha$ the angle between $B$ and $v$ ) is indeed considerably smaller than one. For ASDEX Upgrade a rough estimate gives a ratio of about 0.3. However, the increasing accuracy of the method determining the spectral splitting requires a reconsideration of this issue. Since the Zeeman Effect also linearly depends on $|\boldsymbol{B}|$ it is not expected that measurements of magnetic field changes are affected. However, for the determination absolute values of $|\boldsymbol{B}|$ the influence of the Zeeman Effect is significant.

\subsection{Physical properties derived from MSE spectroscopy}

The two basic measurements of MSE spectroscopy are that of the line splitting and of the ratio of $\sigma$ - to $\pi$-components. In addition, the Doppler shift of the different energy components reveals information about the radial position of the observation volume and the line broadening can be used to infer the beam divergence ${ }^{26,27}$.

Assuming that the details of the beam geometry and beam velocity distribution are known, the line splitting can be used to derive the total magnetic field, $|\boldsymbol{B}|$. At JET this was implemented for the first time, inferring the diamagnetic reduction of the magnetic field due to ion cyclotron resonance heating ${ }^{23}$. Since the associated $\beta$-changes are of the order of a few percent, the required sensitivity must be in the per mill range. Knowing the pressure profile from kinetic measurements of plasma density and temperature, this technique can be used to infer the fast ion pressure contribution to the total pressure. Other early measurements also showed that assuming the toroidal field is known - the inferred radial poloidal field distribution is in agreement with equilibrium calculations which use edge magnetic flux measurements as a constraint $^{18}$. The data analysis originally employed a multi-Gaussian fit to the MSE spectrum using the coupling of parameters such as wavelength splitting and line ratios or their symmetries to minimize the free parameters as far as possible ${ }^{18}$. Recently a forward model has been developed for the inference of the plasma parameters which also includes effects such as the fast ion contribution to the spectrum ${ }^{22,30}$.

The $\sigma$ - to $\pi$-line ratios can be utilized to recover the orientation of the magnetic field, $B_{p o l} / B_{t o r}$, and from that quantities such as the current or safety factor profile ${ }^{29}$. Combining the $\sigma$ - to $\pi$-ratio and the line splitting allows to self-consistently derive direction $\left(B_{p o l} / B_{t o r}\right)$ and magnitude $(|\boldsymbol{B}|)$ of the magnetic field ${ }^{24,28,12}$. Depending on the details of the measurement, special care has to be taken with the polarization dependent reflectivity of mirrors and the population density of the atomic levels. Both can significantly influence the outcome of the $\sigma$ to $\pi$-ratio line ratio measurement. Depending on the mirror properties and the alignment between $\sigma$ - to $\pi$-polarization directions and $p$ - and s-axes of the mirror a large modification of the line ratio measurement can be produced which needs careful calibration ${ }^{12}$. A non-statistical population of the atomic levels introduces a density dependence of the line ratios which has to be included in the analysis of the spectral data ${ }^{12,30}$. If the spectral resolution permits to resolve the Stark lines individually, the $\sigma \pm 1$ to $\pi \pm 3$ line ratios offer the possibility of a measurement independent of the relative population densities, as these transitions originate from the same upper level ${ }^{18,28}$. Some diagnostic applications inject the beam into the plasma vessel filled with neutral gas to calibrate the line ratios in the presence of a known magnetic field without plasma and determine the influence of optical components, e.g. a possible mirror, on the 
measurement ${ }^{28,12}$. In tokamaks the vacuum field without plasma is the toroidal magnetic field which has a radial dependence $\sim 1 / \mathrm{R}$ (where $\mathrm{R}$ is the major radius of the torus). In stellarators the vacuum magnetic field already exhibits a rotational transform and can be calculated from the currents in the magnetic field coils. For beam into gas injection the question about nonstatistical population distribution is even more important ${ }^{25}$. While it has been reported that this calibration method in principle works ${ }^{12}$, large discrepancies between predicted and measured $\sigma$ to $\pi$-ratios have been observed too ${ }^{25}$.

Basically following the calculations of Souw and Uhlenbusch ${ }^{4}$, the combination of Motional Stark and Zeeman Effects has been revisited. Solving the Schrödinger equation in the strong magnetic field limit and neglecting the spin-orbit coupling, but including a Stark term, the modification of the wavelength splitting has been calculated. Generally, the wavelength splitting increases with respect to the pure Stark case. As a result, the apparent value of $|\boldsymbol{B}|$, assuming Stark Effect only, is larger than the value inferred from the combination of Zeeman and Stark Effects. Depending on beam energy, the discrepancies for ASDEX Upgrade beam parameters and a magnetic field of $2.2 \mathrm{~T}$ are between $3 \%$ (for $20 \mathrm{keV}$ deuterium; third energy component) and $1 \%$ (for $60 \mathrm{keV}$ deuterium; full energy component). For an accurate measurement of the absolute magnetic field value this is significant.

\section{Measurement of the polarized line emission}

\subsection{Measurement techniques}

As outlined above the spectral lines of the MSE multiplet are polarized. Rather than utilizing the different emission characteristics of the $\sigma$ - and $\pi$-lines, MSE polarimetry tries to measure their polarization directions directly. The polarization measurements have the principle advantage that they do not depend measurably on the population distribution of the atomic levels. Up to now four measurement techniques have been investigated.

The simplest technique combines two static beam splitting polarizers dividing the incoming light of each line of sight into two polarization components. For each polarization component the full MSE spectrum is recorded ${ }^{18}$. A multi-Gaussian fit is used to separate $\sigma$ - and $\pi$-lines and subtract the unpolarized background signal. A half-wave plate in front of the light collecting optics makes sure that the incoming linearly polarization is approximately split into equal parts. As a result, the ratio of the $\pi$-emission from the two polarization components is a direct measure of the orientation of $\boldsymbol{E}_{\boldsymbol{L}}$ and subsequently $\boldsymbol{B}_{p o l} / \boldsymbol{B}_{t o r}{ }^{31}$. This technique has the advantage that the polarization can be measured while the full spectral information is retained. However, the requirement to record two Stark spectra for each line of sight with sufficient spectral resolution, including a meaningful background measurement, limits the possible time resolution. In addition, the quality of the spectral analysis necessary to separate $\pi$ - from $\sigma$ emission and the unpolarized background determines the sensitivity of the diagnostic to $\boldsymbol{E}_{\boldsymbol{L}}$ variations as it is the case also for the measurement of the $\sigma$ - and $\pi$-line ratios.

A technique proposed by Voslamber ${ }^{32}$ overcomes the last problem. Instead of only using two static linear polarizers this technique uses three linear polarizers and one circular polarizer for each line of sight, which are aligned in such a way that the measured signal corresponds to the four Stokes parameters. Without the necessity of a sophisticated spectral analysis, these four parameters not only contain the information about the polarization of the MSE emission, but 
also can be used to recover the influence of polarizing elements in the transmission optics, such as a non-ideal mirror, without any extra calibration. To be sufficiently sensitive the measurement only requires a spectral resolution which separates $\pi$ - from $\sigma$-emission. However, additional effort is generated by the necessity to record up to four spectra per line of sight. An implementation of this technique in a slightly modified form has been installed and tested on the Large Helical Device (LHD) ${ }^{11}$ measuring small variations of the rotational transform induced by neutral beam current drive.

The today most commonly used technique was first introduced on the tokamak PBX-M $\mathrm{M}^{6}$. The polarimeter consists of two so-called photo-elastic modulators (PEMs) ${ }^{33}$. A PEM generates a time dependent phase modulation by oscillating stress induced birefringence. The MSE polarimeter setup consists of two PEMs at different orientations with respect to their optical axes of birefringence and operating at slightly different frequencies. Typical frequencies of the PEMs used for the MSE polarimeters are 20 and $23 \mathrm{kHz}$. Adjusting the PEM amplitudes in such a way that the maximum phase shift corresponds $\lambda / 2$, they work in a similar way as rotating half-wave plates. Followed by a linear polarizer, the whole assembly transforms any incoming linear polarization into an amplitude modulated signal with the following time response:

$I \propto \sin (2 \gamma) \cos \left(2 \omega_{2} t\right)-\cos (2 \gamma) \cos \left(2 \omega_{1} t\right)+\ldots$, where $\gamma$ is the incoming polarization angle and $\omega_{1}$ and $\omega_{2}$ are the two frequencies of the PEMs ${ }^{6}$. Taking the ratio of the two $\cos \left(2 \omega_{i} t\right)$ terms, $\tan (2 \gamma)$ can be recovered. The intensities at the second harmonic frequency of the PEMs can be measured either by lock-in amplifiers driven by the PEM frequencies or recording the full time evolution of the modulated signal. Subsequently, the modulation intensities at $2 \omega_{i} t$ are inferred by Fourier transforming the measured signal.

In contrast to the first two techniques using static polarizers, the PEM-based dynamic polarimeter relies on the spectral filters to select either the $\sigma$ - or the $\pi$-components of the spectrum. Usually the $\sigma$-component is selected as its central wavelength position only depends on the angle between line of sight and neutral beam, which determines the Doppler shift, but not on the magnetic field strength. For beam energies between $50-100 \mathrm{keV}$ and magnetic fields between $2-3 \mathrm{~T}$ the width of the spectral filters must be in the range of $2-3 \AA$, which requires temperature control to keep the filter at the desired wavelength. Only in spherical tokamaks with their smaller magnetic fields narrower filters have to be used ${ }^{34,35}$. Since the Doppler shift causes the central wavelength of each Stark multiplet to differ for different lines of sight, each line of sight requires a different filter. PEM-based multi-channel MSE polarimeters have been installed on many tokamaks ${ }^{36,37,38,39,40,34,35,41,42}$.

Crucial for any MSE polarimeter is the absolute accuracy of the polarization angle measurement and the calibration to achieve this ${ }^{43}$. Issues involved with the calibration of the polarimeter setups are the Faraday rotation induced by the magnetic field inside the observation optics, reflections from (non-ideal) mirrors, any type of spurious birefringence in the observation optics, or coatings of first mirrors or windows from plasma vessel conditioning or from plasma operation. If the orientation of the optical axis of the observation optics is strictly radial with respect to the magnetic field, Faraday rotation does not occur. However, the optics are usually between the toroidal field coils where the corresponding field is radial and very inhomogeneous. Further out the radial field from the poloidal field coils can also be significant. For most optical setups the optical axis exhibits at least a small component in the direction of the magnetic field. Special glass with a low Verdet constant can be used to minimize the effect ${ }^{44}$. 
In doing so, typical values of the Faraday rotation of the ASDEX Upgrade MSE polarimeter are of the order of $1 \%$ T. In the case of DIII-D even lower values have been achieved ${ }^{45}$. In order to minimize the influence of the mirror on the polarization measurement, in ASDEX Upgrade the mirror is provided with a dielectric coating which for the wavelength range and the angular range given maximizes the reflectivity ${ }^{46}$. In addition, in ASDEX Upgrade the mirror is protected against coatings by an exchangeable glass window in front of the mirror. In fact, thin deposits were found on this window, which could serve as a possible explanation for observed drifts of the offset calibration of the polarization angles.

Comparing the independently measured polarization angles of the $\sigma$ - and $\pi$-components by tuning the wavelength filters to the corresponding wavelengths a dependence of the difference of the $\sigma$ - and $\pi$-polarization angles on the plasma density has been found on ASDEX Upgrade. Without any modification of the polarization angles or other spurious polarization contributions this difference should be $90^{\circ}$. However, above line integrated densities of about $6 \times 10^{19} \mathrm{~m}^{-3}$ deviations from this value of up to $15^{\circ}$ are observed. While the reason is still unclear, this behaviour seems to coincide with the introduction of tungsten as a plasma facing material. The question is whether polarized plasma radiation lies within the wavelength band of the polarimeter or whether the wall reflections turn unpolarized background radiation, e.g. bremsstrahlung, into partially polarized light ${ }^{16}$. Measurements of the wall-reflections inside Alcator C-Mod with an unpolarized light source indeed show a large polarization fraction favouring the second explanation. Also beam modulation experiments with plasma show a polarization signal during the beam-off times ${ }^{44}$.

\subsection{Physical properties derived from MSE polarimetry}

In tokamaks the main objective of the polarimeter systems is to measure the poloidal magnetic field distribution or the current density profile which are crucial quantities determining confinement and stability. In stellarators (or heliotrons) the quantities of interest are currents driven by heating systems or bootstrap currents. To test physical models, both, the measurements in tokamaks and stellarators require a very high absolute accuracy typically corresponding to magnetic field pitch angle $\left(\arctan \left(B_{p o} / B_{t o r}\right)\right)$ errors between $0.5^{\circ}$ and $0.1^{\circ}$. Considering all possible error sources the lower value maybe at the limit what is technically achievable.

A famous example is the sawtooth instability occurring in tokamaks and related to this the formation and possible reconnection of internal magnetic islands. Although regularly observed, a conclusive theoretical model does not exist until today. Only recently theoretical advances give an explanation for the fast reconnection time observed in tokamaks ${ }^{47}$. Closely related to the reconnection process is the behaviour of the safety factor profile. Crucial for the understanding is the temporal evolution of the safety factor at the centre of the plasma, $q_{0}$, and whether it drops significantly below 1 or rises above 1 just after the sawtooth crash. However, up to now the experimental evidence is inconsistent supporting both, full reconnection $\left(q_{0} \text { rises above } 1\right)^{48}$ or partial reconnection $\left(q_{0} \text { stays below } 1\right)^{49,50}$.

Another important physical quantity which can be derived from an MSE polarimetry measurement is the radial electric field, $\boldsymbol{E}_{r}^{51,52,40}$. In tokamaks and stellarators the radial electric field plays a central role in the plasma transport and in the understanding of confinement transitions. Despite the small value of $\left|\boldsymbol{E}_{r}\right|, \boldsymbol{E}_{r}$ can make significant contribution to the measured 
orientation of $\boldsymbol{E}_{L}+\boldsymbol{E}_{r}$, in particular because for the usual MSE diagnostic setups $\boldsymbol{E}_{r}$ is approximately orthogonal to $\boldsymbol{E}_{L}$. One possible way to extract $\boldsymbol{E}_{r}$ from the polarization measurement is to utilize the first and the second energy component simultaneously thus introducing another independent measurement of the polarization angle with different beam velocity values. Another possibility is to employ two differently oriented beams at the same beam energy, using the fact that now the observation angles between line of sight and the neutral beams are different.

Nowadays, many tokamak applications use MSE data as a constraint for equilibrium codes. Thereby, the measured polarization angles serve as a strong constraint for the current density profile ${ }^{53}$. Using this approach, also other quantities can be derived. For instance, the non-inductive plasma current profile can be determined by computing the temporal evolution of the poloidal flux profile ${ }^{54}$ derived from such equilibrium calculations.

\section{Imaging MSE}

Imaging MSE refers to a new technique which up to now has been tested on TEXTOR ${ }^{55}$, $\mathrm{KSTAR}^{42}$ and ASDEX Upgrade ${ }^{56},{ }^{57},{ }^{58}$. The system works like a static polarimeter capable of producing a full 2-dimensional image of the polarized plasma emission ${ }^{59}$. It consists of a conventional imaging system with a CCD or CMOS type detector. A wavelength filter is chosen in such a way that a large fraction of the MSE spectrum ( $\sigma$ - and $\pi$-components and also parts of second or third energy component) is transmitted, while the edge Balmer- $\alpha$ is blocked out. Birefringent plates, introduced in the optical path, generate a wavelength and polarization dependent phase shift transforming the incoming polarization information into an interference pattern: $I \propto 1+\zeta \cos (2 \gamma) \cos \left(\omega_{x} x\right)+\zeta \sin (2 \gamma) \cos \left(\omega_{x} x+\omega_{y} y\right)+\zeta \sin (2 \gamma) \cos \left(\omega_{x} x-\omega_{y} y\right)$, where $\mathrm{I}$ is the measured intensity as function of the image coordinates $x$ and $y, \omega_{x}$ and $\omega_{y}$ are approximately constant, and $\zeta$ is the so-called spectral contrast, which is a slowly varying function depending on the optical properties of the birefringent plates and spectral distribution of the incoming light. Fourier-transforming the image, $I$, and separating the components yields the polarization angle $\gamma$ (projection of the orientation of $\boldsymbol{E}_{L}$ onto a plane perpendicular the viewing direction) as a function of the image coordinates. An example of an ASDEX Upgrade measurement ${ }^{57}$ is illustrated in Fig. 3.

A clear advantage of the Imaging MSE technique is the wealth of information gained by recording emission images and at the same time having a 2-dimensional measurement of the polarization angles. Since in contrast to the PEM-based polarimeter the bigger part of the spectrum is used, the signal to noise ratio is very good. Typical filters cover all spectral lines of the full energy components and parts of the second and even third energy component and therefore have a width which is about an order of magnitude broader than that required for MSE polarimetry. The imaging technique allows observing different beams or more than one beam simultaneously. In addition background images of e.g. the plasma vessel walls can be recorded. Together with the beam emission image this defines the viewing geometry. However, to get away from plasma dependent calibrations and achieving the desired accuracy requires an understanding of all polarimeter details, such as the behaviour of the spectral contrast which is assumed to exactly cancel when taking the ratio of the Fourier components. Issues which remain also for an Imaging MSE diagnostic are the Faraday rotation calibration or the possible effects 
of mirrors or spurious birefringence from the optical elements such as vacuum windows. Another advantage of an Imaging MSE system is that it is not affected by a spectrally broadband polarized emission which is suspected to influence PEM-based systems. Polarization effects from narrow band plasma emission in the passband would be apparent as a disturbance to the expected Doppler phase shift and could be masked during the signal processing.
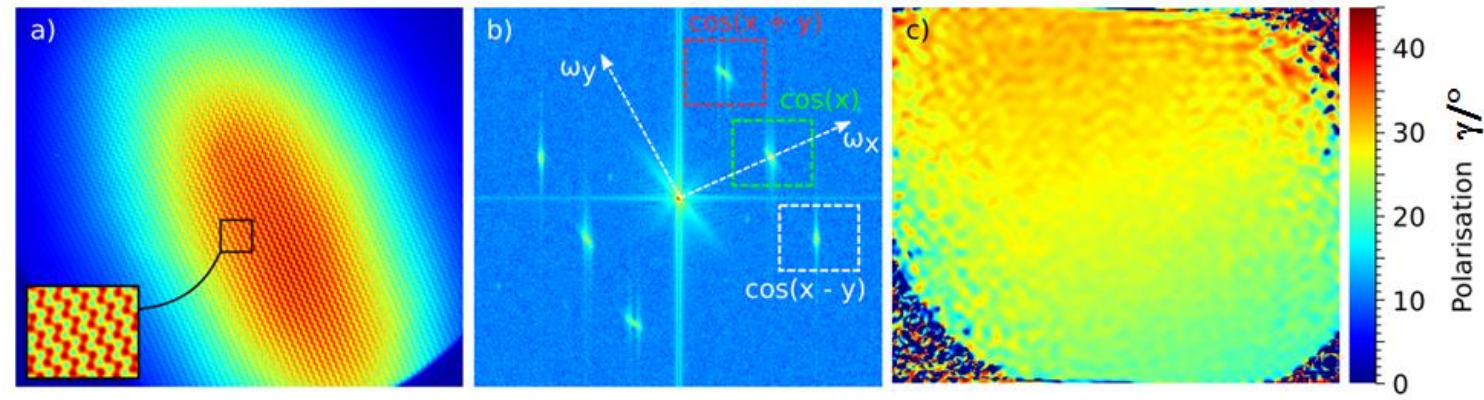

Fig. 3a) shows the beam emission image, superimposed with the interference pattern. The Fourier transform, $b$ ), exhibits the three components which contain the information about the polarization angle. c) Plotting the polarization angle as a function of $x$ and y yields a 2dimensional polarization distribution.

With the ASDEX Upgrade prototype Imaging MSE system good agreement of the measurements with both, theoretical predictions and the PEM-based MSE polarimeter has been achieved $^{58}$. The time resolution of $5 \mathrm{~ms}$ allows resolving sawtooth oscillations. Preliminary analysis shows that the expected current density dynamics within the plasma core are well resolved, although the q-values are still subject to obtaining a good absolute calibration.

\section{Conclusions}

The Motional-Stark-Effect is the most widely used technique to gain information about the internal magnetic field distribution and dynamics of magnetic fusion experiments. Measurements of the full MSE spectrum can be used to derive both the magnetic field strength and its orientation. Aiming at absolute accuracies of $|\boldsymbol{B}|$ of the order of $0.1 \%$, the influence of the Zeeman Effect has to be considered. $\boldsymbol{B} /|\boldsymbol{B}|$ can be inferred from a line ratio measurement which either relies on the knowledge of the details of the population density distribution or a very good spectral resolution to accurately resolve the individual line intensities of the Stark multiplet. Polarimeters make use of the polarization information contained in the Stark emission to derive $\boldsymbol{B} /|\boldsymbol{B}|$. The most commonly used technique is a dynamic PEM-based polarimeter which combines high time resolution with a comparatively simple data analysis. Imaging MSE systems have been introduced recently showing significant advantages. However, the full understanding of these systems is still progressing. After successfully testing a prototype on ASDEX Upgrade, a dedicated Imaging MSE diagnostic has been developed and installed. First results are expected this year. However, all polarimeter techniques, need elaborate calibration techniques to achieve the necessary accuracy. 
A frequently asked question is how to implement an MSE diagnostic on ITER. Studies propose both a spectroscopic system ${ }^{60}$ and a polarimeter ${ }^{61}$. As was investigated earlier ${ }^{18}$, the first study suggests using the measurement of the line splitting to derive the tokamak q-profile thus avoiding detrimental effects of the large number of mirrors required to protect the vacuum window and the subsequent optical elements against neutron radiation. It would be certainly interesting to investigate whether an Imaging MSE diagnostic would solve some of the issues related to a polarimetric measurement on ITER.

\section{References}

[1] H. Soltwisch, Plasma Phys. Control. Fusion 34 (1992) 1669

[2] R. Jaenicke et al., Nucl. Fusion 33 (1993) 687

[3] C. Breton, C. D. Michelis, M. Finkenthal and M. Mattiolo, J. Phys. B: Atom. Molec. Phys. 13 (1980) 1703

[4] E. K. Souw and J. Uhlenbusch, Physica 122C (1983) 353

[5] R. C. Isler, Phys. Rev. A 14 (1976) 1015

[6] F. M. Levinton et al., Phys. Rev. Lett. 63 (1989) 2060

[7] A. Boileau et al., J. Phys. B: At. Mol. Opt. Phys. 22 (1989) L145

[8] C. T. Holcomb et al., Rev. Sci Instrum. 77 (2006) 10E506

[9] N. C. Hawkes et al., Rev. Sci. Instrum. 70 (1999) 894

[10] F. M. Levinton et al., Rev. Sci. Instrum. 79 (2008) 10F522

[11] K. Ida et al., Rev. Sci Instrum. 76 (2005) 053505

[12] K. J. McCarthy et al., Contrib. Plasma Phys. (2015) DOI 10.1002/ctpp.201400067

[13] E. Delabie et al., Plasma Phys. Control. Fusion 52 (2010) 125008

[14] O. Marchuk, Yu Ralchenko, and D. R. Schulz, Plasma Phys. Control Fusion 54 (2012) 095010

[15] H. Y.-H. Yuh, The Motional Stark Effect Diagnostic on Alcator C-Mod, PhD Thesis, MIT (2005) http://hdl.handle.net/1721.1/34976

[16] R. T. Mumgaard, S.D. Scott, and R.S. Granetz, Toward Reactor Relevant MSE: In-situ Calibration and Polarized Background Subtraction on Alcator C-Mod, presented at the $20^{\text {th }}$ Topical Conference on High-Temperature Plasma Diagnostics, Atlanta, USA (2014)

[17] R. K. Janev, C. D. Boley and D. E. Post, Nucl. Fusion 29 (1989) 2125

[18] W. Mandl, R. C. Wolf, M. G. von Hellermann and H. P. Summers, Plasma Phys. Control Fusion 35 (1993) 1373

[19] Original from www.jet.efda.org, similar picture available at www.euro-fusion.org

[20] A. Dinklage et al., Fusion Sci. Techn. 59 (2011) 406

[21] R. C. Wolf et al., Nucl. Fusion 41 (2001) 1259

[22] R. Reimer et al., Contrib. Plasma Phys. 50 (2010) 731

[23] R. C. Wolf et al., Nucl. Fusion 33 (1993) 1835

[24] N. Pablant et al., Rev. Sci. Instrum. 79 (2008) 10F517

[25] M. F. Gu, C. T. Holcomb,R. J. Jayakuma, and S. L. Allen, J. Phys. B:At. Mol. Opt. Phys. 41 (2008) 095701 
[26] R. C. Wolf, D. Ciric and R. W. T. König, JET Report (1994) JET-P(94)68, www.eurofusionscipub.org/archives/jet-archive/in-situ-determination-of-the-jet-neutral-heating-beamdivergence-by-beam-emission-spectroscopy

[27] M. G. von Hellermann et al., AIP Conf. Proc. 1058 (2008) 187

[28] N. Pablant et al., Rev. Sci. Instrum. 81 (2010) 10D729

[29] K. Jakubowska et al., Rev. Sci. Instrum. 75 (2004) 3475

[30] R. Reimer et al., Rev. Sci Instrum. 84 (2013) 113503

[31] R.C. Wolf, J. O'Rourke, A.W. Edwards and M. Von Hellermann, Nucl. Fusion 33 (1993) 663

[32] D. Voslamber, Rev. Sci. Instrum. 66 (1995) 2892

[33] J. C. Kemp, J. Opt. Soc. Am. 59 (1969) 950

[34] F. M. Levinton and H. Yuh, Rev. Sci. Instrum. 79 (2008) 10F522

[35] N. J. Conway et al., Rev. Sci. Instrum. 81 (2010) 10 D738

[36] F. M. Levinton, Rev. Sci. Instrum. 63 (1992) 5157

[37] D. Wróblewski, K. H. Burrell, L. L. Lao, P. Politzer, and W. P. West, Rev. Sci. Instrum. 61 (1990) 3552

[38] C. T. Holcomb et al., Rev. Sci. Instrum. 77 (2006) 10 E506

[39] N. C. Hawkes and M. Brix, Rev. Sci. Instrum. 77 (2006) 10E509

[40] J. Hobirk et al., Measurements of the Poloidal Magnetic and Radial Electric Field Profiles in ASDEX Upgrade and JET, Proceedings of the International Conference on Advanced Diagnostics for Magnetic and Inertial Fusion, September 2001 at Vllla Monastero, Varenna, Italy, Springer 2002, DOI 10.1007/978-1-4419-8696-2

[41] J. Ko, S. Scott, M. Bitter, and S. Lerner, Rev. Sci. Instrum. 79 (2008) 10F520

[42] J. Chung et al., J. Korean Phys. Soc. 65 (2014) 1257

[43] R. T Mumgaard, S. D. Scott, and J. Ko, Rev. Sci. Instrum. 85 (2014) 053505

[44] B. W. Rice, D. G. Nilson. and D. Wróblewski, Rev. Sci. Instrum. 66 (1995) 373

[45] B. W. Rice, Fusion Eng. and Design 34 (1997) 135

[46] R. C. Wolf et al., Europhysics Conference Abstracts (Proc. of the 24th EPS Conference on Controlled Fusion and Plasma Physics, Berchtesgaden, 1997), Vol. 21A, part IV (1997) 1509

[47] S. Günter, Q. Yu, K Lackner, A. Bhattacharjee. and Y-M Huang, Plasma Phys. Control. Fusion 57 (2015) 014017

[48] D. Wroblewski and R. T. Snider, Phys. Rev. Lett. 71 (1993) 859

[49] M. Yamada et al., Review of Scientific Instruments 63 (1992) 4623

[50] R. Koslowski, Fusion Sci. and Techn. 47 (2005) 260

[51] B. W. Rice, K. H. Burrell, L. L. Lao, and Y. R. Lin-Liu, Phys. Rev. Lett. 79 (1997) 2694

[52] M. C. Zarnstorff, F. M. Levinton, S. H. Batha, and E. J. Synakowski, Phys. Plasmas 4 (1997) 1097

[53] L. L. Lao et al., Fusion Sci. And Techn. 48 (2005) 968

[54] C. B. Forest et al., Phys. Rev. Lett. 73 (1994) 2444

[55] J. Howard, A. Diallo, R. Jaspers, and J. Chung, Plasma and Fusion Research 5 (2010) S1010

[56] O. Ford, J. Howard, and R. C. Wolf, Proc. 40 ${ }^{\text {th }}$ EPS Conference on Plasma Physics, Helsinki (2013), P6.006 (ocs.ciemat.es/EPS2013PAP/pdf/P6.006.pdf) 
[57] O. Ford et al., Proc. 40 ${ }^{\text {th }}$ EPS Conference on Plasma Physics, Helsinki (2013), O2.110.pdf (ocs.ciemat.es/EPS2013PAP/pdf/O2.110.pdf)

[58] O. P. Ford et al., J. Howard, and R. C. Wolf, The ASDEX Upgrade Prototype Imaging Motional Stark Effect (IMSE) Diagnostic, to be submitted to Rev. Sci. Instrum. (2015)

[59] J. Howard, J. Phys. B: At. Mol. Opt. Phys. 43 (2010) 144010

[60] E. L. Foley, F. M. Levinton, H. Y. Yuh, and L. E. Zakharov, Rev. Sci. Instrum. 79 (2008) 10F521

[61] M. A. Makowski et al., Rev. Sci. Instrum. 79 (2008) 10F519 